Original Article (short paper)

\title{
Do muscular strength and jump power tests reflect the effectiveness of training programs for basketball athletes?
}

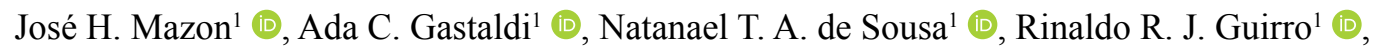 \\ Victor B. Ribeiro $^{1}$ (), Tábata P. Facioli ${ }^{1}$ (]), Stella V. Philbois ${ }^{1}$ (), Hugo C. D. Souza ${ }^{1}$ (]) \\ ${ }^{1}$ Universidade de São Paulo, USP, Exercise Physiology Laboratory, Department of Rehabilitation \\ and Functional Performance, Ribeirão Preto Medical School, Riberião Preto, SP, Brazil
}

\begin{abstract}
Aims: Muscular strength (MS) and jump power (JP) tests are used to assess athletic ability and measure the effectiveness of training programs. However, their use in various sport modalities needs to be investigated further. This study aimed to explore the changes in MS and JP during three different moments of a macrocycle training session and verify the validity of the tests used to predict the effectiveness of basketball training programs. Methods: During macrocycle training (three different moments), sixteen basketball players were evaluated for MS (measured using isokinetic dynamometry at the speed of $60^{\circ} / \mathrm{s}$ ) during concentric contraction of knee flexor and extensor muscles and JP, using countermovement vertical jump (CMVJ) on a force platform. Results: Peak torque and maximal work values for knee extension and flexion showed no differences, during the three moments analyzed. Additionally, no changes were observed for CMVJ. Conclusions: Our results suggest that the effectiveness of basketball training programs does not seem to be related to the performance achieved by athletes on the tests used. Moreover, the lack of changes in MS and JP values during the macrocycle could be related to the training structure used; volume, intensity, density and workload specificity.
\end{abstract}

Keywords: team sport; performance; measurement

\section{Introduction}

Basketball is an intermittent sport modality with a significant anaerobic metabolism demand. In fact, this metabolic demand is necessary for supporting the performance of moderate and highintensity movements and periods of active and passive recovery ${ }^{1,2}$. Additionally, some specific attributes of basketball, such as changes in direction, acceleration, speed and jump ability, are closely related to muscle power ${ }^{3}$. Thus, the neuromuscular status of the athlete, which includes strength and power abilities, as well as the different uses of these during participation in a game, may have an effect on the athletic performance of this sport modality ${ }^{4,5}$.

In the context, the efficacy of the programs and methods utilized in basketball training is assessed through the athletes performance, using various physical tests ${ }^{6,7}$. However, it is fundamentally important that each test is selected according to the specific characteristics of the sport training modality. Many ongoing studies are focused on elucidation of these critical issues. In basketball athletes, evaluation of muscular strength (MS) and jump power (JP) abilities has been used to monitor fatigue and performance, as well as identify muscle imbalances and asymmetries ${ }^{7-9}$. However, the relationship between MS and $\mathrm{JP}$ values and the athletes' performance in basketball is not fully understood and needs further investigation. Moreover, the protocols used to evaluate basketball skills, measure abilities of power and strength in many different ways, which makes it difficult for choosing the most adequate one ${ }^{3}$.

In fact, little is known about the applicability of the results obtained from certain physical tests used to assess specific athletic performance in team sports. Furthermore, it has been shown that maximal strength and jump power abilities are not used very often during a basketball game ${ }^{10}$. Therefore, this study aimed to explore the changes in MS and JP, during three different moments of a macrocycle training session and verify the validity of the tests used to predict the effectiveness of basketball training programs. We hypothesize that there will be changes in strength and power jump of athletes during macrocycle training and the tests selected to evaluate these physical abilities will be able to identify possible alterations promoted by this specific basketball training program.

\section{Methods}

\section{Participants}

16 male basketball players (mean \pm SD: age, $18.3 \pm 0.7$ years; height, $188 \pm 9.98 \mathrm{~cm}$; weight, $85.6 \pm 12.1 \mathrm{~kg}$; training history, $3 \pm 0.9 \mathrm{y}$ ), who were registered in the state championship, participated in the study. The exclusion criteria were as follows: a) showing musculoskeletal injuries that would prevent the athlete from performing the tests used; b) not participate in training and competitions.

The participants were screened in the Exercise Physiology Laboratory, Department of Rehabilitation and Functional Performance, Ribeirão Preto Medical School, University of São Paulo, Brazil. The study protocol followed the code of ethics of the World Medical Association (Declaration of Helsinki), 
published in the British Medical Journal (1964) and was approved by the Human Research Ethics Committee of "Hospital das Clinicas" of the Ribeirão Preto Medical School, University of São Paulo, Brazil. All participants signed an informed consent form prior to the start of the study (Protocol: 162.079/2013)

\section{Procedures}

The duration of the macrocycle training was 34 weeks and the composition of the microcycle training was established as shown in Table 1. The technical-tactical training intensity was quantified in arbitrary units, using the method proposed by Edwards ${ }^{12}$. This is a (heart-rate-based method that integrates the total volume of the training session with the total intensity of the exercise session, relative to five intensity zones (Fig 1). The strength and power training protocols are shown in Tables 2 and 3 . Table 2 shows the distribution of the resistance training load, performed in the fitness center, as training volume (number of sessions a week, number of combinations and repetitions for each exercise) and intensity (\% of maximal repetition), during the 34 weeks of the macrocycle.

Table 1. Composition of training microcycles

\begin{tabular}{|c|c|c|c|c|c|c|c|}
\hline Microcycles & $\begin{array}{l}\text { Total number } \\
\text { of days }\end{array}$ & $\begin{array}{l}\text { Number of } \\
\text { sessions }\end{array}$ & $\begin{array}{l}\text { Technical-tactical } \\
\text { sessions }\end{array}$ & $\begin{array}{c}\text { Physical training } \\
\text { sessions }\end{array}$ & $\begin{array}{l}\text { Total duration } \\
\text { (min) }\end{array}$ & $\begin{array}{l}\text { Physical } \\
\text { evaluations }\end{array}$ & $\begin{array}{l}\text { Official } \\
\text { games }\end{array}$ \\
\hline 1 & 2 & 4 & 3 & 1 & 360 & --- & --- \\
\hline 2 & 6 & 11 & 5 & --- & --- & 6 & --- \\
\hline 3 & 6 & 10 & 8 & 3 & 900 & --- & --- \\
\hline 4 & 6 & 11 & 8 & 3 & 990 & --- & --- \\
\hline 5 & 6 & 10 & 7 & 3 & 900 & --- & --- \\
\hline 6 & 6 & 10 & 7 & 3 & 900 & --- & 1 \\
\hline 7 & 6 & 10 & 7 & 3 & 900 & --- & --- \\
\hline 8 & 6 & 9 & 6 & 2 & 810 & --- & 2 \\
\hline 9 & 6 & 11 & 8 & 5 & 990 & --- & --- \\
\hline 10 & 6 & 10 & 7 & 5 & 900 & --- & 1 \\
\hline 11 & 6 & 10 & 7 & 3 & 900 & --- & 1 \\
\hline 12 & 6 & 10 & 7 & 3 & 900 & --- & 1 \\
\hline 13 & 6 & 10 & 7 & 3 & 900 & --- & 1 \\
\hline 14 & 6 & 10 & 7 & 3 & 900 & --- & 1 \\
\hline 15 & 5 & 9 & 6 & 2 & 810 & --- & 2 \\
\hline 16 & 5 & 9 & 4 & --- & --- & 6 & --- \\
\hline 17 & 6 & 11 & 8 & 3 & 990 & --- & --- \\
\hline 18 & 6 & 10 & 7 & 3 & 900 & --- & 1 \\
\hline 19 & 6 & 11 & 8 & 5 & 990 & --- & --- \\
\hline 20 & 6 & 10 & 7 & 5 & 900 & --- & --- \\
\hline 21 & 7 & 9 & 6 & 2 & 810 & --- & 2 \\
\hline 22 & 4 & 6 & 3 & 2 & 540 & --- & 2 \\
\hline 23 & 6 & 10 & 7 & 5 & 900 & --- & --- \\
\hline 24 & 6 & 11 & 8 & 5 & 990 & --- & --- \\
\hline 25 & 6 & 10 & 7 & 3 & 900 & --- & 1 \\
\hline 26 & 6 & 10 & 7 & 3 & 900 & --- & 1 \\
\hline 27 & 6 & 10 & 7 & 3 & 900 & --- & 1 \\
\hline 28 & 6 & 10 & 7 & 3 & 900 & --- & 1 \\
\hline 29 & 5 & 10 & 7 & 3 & 900 & --- & 1 \\
\hline 30 & 6 & 10 & 5 & --- & --- & 6 & --- \\
\hline 31 & 6 & 11 & 8 & 3 & 990 & --- & --- \\
\hline 32 & 6 & 10 & 7 & 2 & 900 & --- & 1 \\
\hline 33 & 4 & 6 & 4 & 1 & 540 & --- & 1 \\
\hline 34 & 4 & 6 & --- & --- & --- & --- & --- \\
\hline
\end{tabular}


Fig. 1 Magnitude of technical-tactical training load in arbitrary units. Values are presented as mean $\pm \mathrm{SD}$.

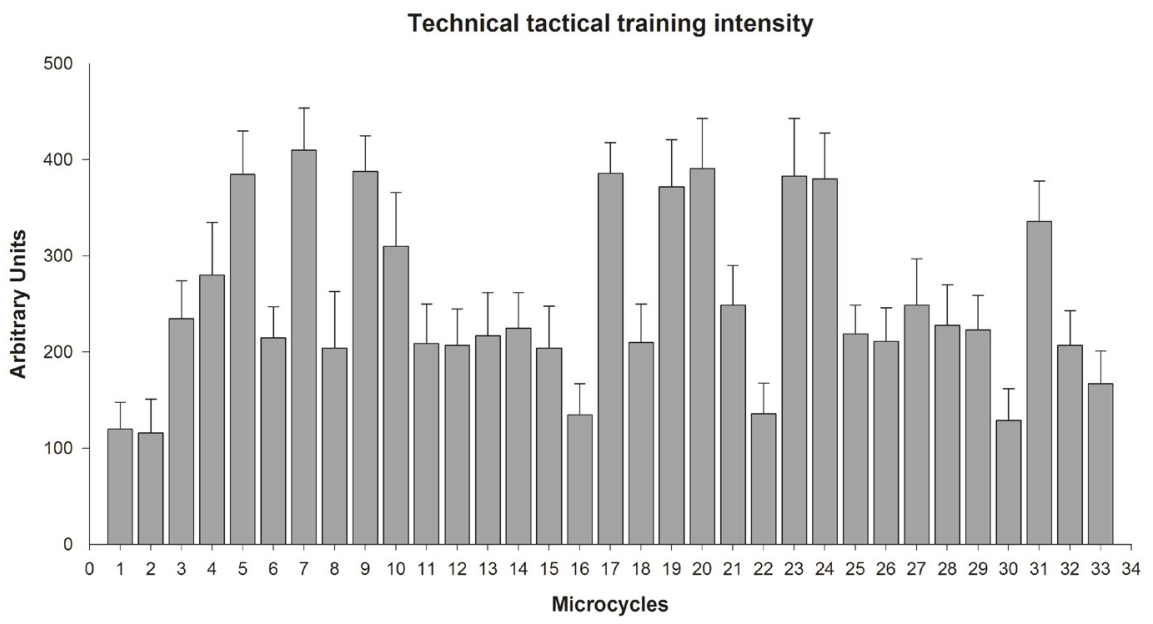

Table 2. Description of strength training

\begin{tabular}{|c|c|c|c|c|c|c|c|c|c|c|c|c|}
\hline \multirow[b]{2}{*}{ Microcycles } & \multicolumn{4}{|c|}{ Volume and intensity } & \multicolumn{8}{|c|}{ Lower limbs exercises and weekly frequency of use } \\
\hline & $\begin{array}{c}\text { Number } \\
\text { of sessions }\end{array}$ & $\begin{array}{c}1 \mathrm{Rm} \\
(\%)\end{array}$ & $\begin{array}{c}\text { Number } \\
\text { of sets }\end{array}$ & $\begin{array}{l}\text { Number of } \\
\text { repetitions }\end{array}$ & $\begin{array}{c}\text { Leg } \\
\text { extension }\end{array}$ & $\begin{array}{l}\text { Leg } \\
\text { curl }\end{array}$ & $\begin{array}{c}\text { Thigh } \\
\text { adductor }\end{array}$ & $\begin{array}{l}\text { Leg } \\
\text { press }\end{array}$ & $\begin{array}{l}\text { Calf } \\
\text { raise }\end{array}$ & Squat & Deadlifting & $\begin{array}{c}\text { Step-up } \\
\text { knee }\end{array}$ \\
\hline 1 & 1 & --- & --- & --- & --- & --- & --- & --- & --- & --- & --- & --- \\
\hline 2 & --- & --- & --- & --- & --- & --- & --- & --- & --- & --- & --- & --- \\
\hline 3 & 3 & 60 & 3 & 12 & 3 & 3 & 3 & --- & 3 & --- & --- & --- \\
\hline 4 & 3 & 70 & 3 & 10 & 3 & 3 & --- & 3 & --- & 3 & --- & --- \\
\hline 5 & 3 & 80 & 4 & 8 & 2 & 2 & --- & 3 & --- & 3 & 1 & 1 \\
\hline 6 & 3 & 80 & 4 & 8 & 1 & 1 & --- & 3 & --- & 3 & 2 & 2 \\
\hline 7 & 3 & 90 & 4 & 6 & --- & --- & --- & 3 & --- & 3 & 3 & 3 \\
\hline 8 & 2 & 70 & 3 & 10 & --- & --- & --- & 2 & --- & 2 & 2 & 2 \\
\hline 9 & 3 & 60 & 3 & 12 & 1 & 1 & 1 & 2 & 1 & 2 & 2 & 2 \\
\hline 10 & 3 & 60 & 3 & 12 & 1 & 1 & 1 & 2 & 1 & 2 & 2 & 2 \\
\hline 11 & 3 & 70 & 3 & 10 & --- & --- & --- & 3 & --- & 3 & 3 & 3 \\
\hline 12 & 3 & 70 & 3 & 10 & 1 & 1 & 1 & 2 & 1 & 2 & 2 & 2 \\
\hline 13 & 3 & 60 & 3 & 12 & --- & --- & --- & 3 & --- & 3 & 3 & 3 \\
\hline 14 & 3 & 70 & 3 & 10 & 1 & 1 & 1 & 2 & 1 & 2 & 2 & 2 \\
\hline 15 & 2 & 60 & 3 & 12 & --- & --- & --- & 2 & --- & 2 & 2 & 2 \\
\hline 16 & --- & --- & --- & --- & --- & --- & --- & --- & --- & --- & --- & --- \\
\hline 17 & 3 & 80 & 4 & 8 & --- & --- & --- & 3 & --- & 3 & 3 & 3 \\
\hline 18 & 3 & 90 & 4 & 6 & --- & --- & --- & 3 & --- & 3 & 3 & 3 \\
\hline 19 & 3 & 70 & 3 & 10 & 1 & 1 & 1 & 2 & 1 & 2 & 2 & 2 \\
\hline 20 & 3 & 60 & 3 & 12 & --- & --- & --- & 3 & --- & 3 & 3 & 3 \\
\hline 21 & 2 & 70 & 3 & 10 & --- & --- & --- & 2 & --- & 2 & 2 & 2 \\
\hline 22 & 2 & 70 & 3 & 10 & --- & --- & --- & 2 & --- & 2 & 2 & 2 \\
\hline 23 & 3 & 70 & 3 & 10 & 1 & 1 & 1 & 2 & 1 & 2 & 2 & 2 \\
\hline 24 & 3 & 60 & 3 & 12 & --- & --- & --- & 3 & --- & 3 & 3 & 3 \\
\hline 25 & 3 & 60 & 3 & 12 & --- & --- & --- & 3 & --- & 3 & 3 & 3 \\
\hline 26 & 3 & 70 & 3 & 10 & --- & --- & --- & 3 & --- & 3 & 3 & 3 \\
\hline 27 & 3 & 60 & 3 & 12 & 1 & 1 & 1 & 2 & 1 & 2 & 2 & 2 \\
\hline 28 & 3 & 70 & 3 & 10 & --- & --- & --- & 3 & --- & 3 & 3 & 3 \\
\hline 29 & 3 & 60 & 3 & 12 & --- & --- & --- & 3 & --- & 3 & 3 & 3 \\
\hline 30 & --- & --- & --- & --- & --- & --- & --- & --- & --- & --- & --- & --- \\
\hline 31 & 3 & 70 & 3 & 10 & --- & --- & --- & 3 & --- & 3 & 3 & 3 \\
\hline 32 & 2 & 60 & 3 & 12 & --- & --- & --- & 2 & --- & 2 & 2 & 2 \\
\hline 33 & 1 & 60 & 3 & 12 & --- & --- & --- & 1 & --- & 1 & 1 & 1 \\
\hline 34 & --- & --- & --- & ---- & --- & --- & --- & --- & --- & --- & --- & --- \\
\hline
\end{tabular}


Table 3. Speed and Power training

\begin{tabular}{|c|c|c|c|c|c|c|c|}
\hline \multirow{2}{*}{ Microcycles } & \multirow{2}{*}{$\begin{array}{l}\text { Resisted sprints and } \\
\text { plyometric training } \\
\text { Number of sessions }\end{array}$} & \multicolumn{3}{|c|}{$\begin{array}{c}\text { Resisted sprints training } \\
\text { (session/volume) }\end{array}$} & \multicolumn{3}{|c|}{$\begin{array}{c}\text { Plyometric training } \\
\text { (exercises and session/volume ) }\end{array}$} \\
\hline & & $\begin{array}{l}\text { Number } \\
\text { of sets }\end{array}$ & $\begin{array}{c}\text { Duration } \\
\text { of set (s) }\end{array}$ & $\begin{array}{c}\text { Interval between } \\
\text { sets (min) }\end{array}$ & Squat jump & $\begin{array}{l}\text { Concentric } \\
\text { box jump }\end{array}$ & $\begin{array}{l}\text { Depth } \\
\text { jump }\end{array}$ \\
\hline 1 & --- & --- & --- & --- & --- & --- & --- \\
\hline 2 & --- & --- & --- & --- & --- & --- & --- \\
\hline 3 & --- & --- & --- & --- & --- & --- & --- \\
\hline 4 & --- & --- & --- & --- & --- & --- & --- \\
\hline 5 & --- & --- & --- & --- & --- & --- & --- \\
\hline 6 & --- & --- & --- & --- & --- & --- & --- \\
\hline 7 & --- & --- & --- & --- & --- & --- & --- \\
\hline 8 & --- & --- & --- & --- & --- & --- & --- \\
\hline 9 & 2 & 9 & 10 & 1 & 24 & 18 & 18 \\
\hline 10 & 2 & 9 & 10 & 1 & 30 & 24 & 24 \\
\hline 11 & --- & --- & --- & --- & --- & --- & --- \\
\hline 12 & --- & --- & --- & --- & --- & --- & --- \\
\hline 13 & --- & --- & --- & --- & --- & --- & --- \\
\hline 14 & --- & --- & --- & --- & --- & --- & --- \\
\hline 15 & --- & --- & --- & --- & --- & --- & --- \\
\hline 16 & --- & --- & --- & --- & --- & --- & --- \\
\hline 17 & --- & --- & --- & --- & --- & --- & --- \\
\hline 18 & --- & --- & --- & --- & --- & --- & --- \\
\hline 19 & 2 & 9 & 10 & 1 & 24 & 18 & 18 \\
\hline 20 & 2 & 9 & 10 & 1 & 30 & 24 & 24 \\
\hline 21 & --- & --- & --- & --- & --- & --- & --- \\
\hline 22 & --- & --- & --- & --- & --- & --- & --- \\
\hline 23 & 2 & 9 & 10 & 1 & 30 & 24 & 24 \\
\hline 24 & 2 & 9 & 10 & 1 & 30 & 24 & 24 \\
\hline 25 & --- & --- & --- & --- & --- & --- & --- \\
\hline 26 & --- & --- & --- & --- & --- & --- & --- \\
\hline 27 & --- & --- & --- & --- & --- & --- & --- \\
\hline 28 & --- & --- & --- & --- & --- & --- & --- \\
\hline 29 & --- & --- & --- & --- & --- & --- & --- \\
\hline 30 & --- & --- & --- & --- & --- & --- & --- \\
\hline 31 & --- & --- & --- & --- & --- & --- & --- \\
\hline 32 & --- & --- & --- & --- & --- & --- & --- \\
\hline 33 & --- & --- & --- & --- & --- & --- & --- \\
\hline 34 & --- & --- & --- & --- & --- & --- & --- \\
\hline
\end{tabular}

The exercises used to develop the lower limb strength, as well as their weekly use frequency, are also included in Table 2 . The volume and intensity of the resistance training oscillate ( 3 to 4 sets for each exercise, with load between 60 and $90 \%$ of a maximum voluntary contraction - 1RM) due to the microcycle characteristics and also the competition schedule. During the competitive period (microcycle 6-33), exercises that required greater specificity of motor gesture 
in relation to the game actions were emphasized. As seen in Table 3, the methods used to develop power jump and speed capability. We respected the principles of individuality and training specificity in the plyometry (power jump) and tracking (speed) sessions. The plyometric jumps and tracking were performed in blocks with submaximal and maximal intensities and all series were followed by specific actions with the ball. The volume of the plyometric training sessions was determined by the average number of jumps performed by the athletes during a basketball game (60 to 78 jumps). Twelve sessions of plyometry were performed, all during the competitive period, due to the competition schedule (short preparation period followed by an extended period of competition), as shown in Tables 1 and 3 .

MS and JP were measured in three different moments during the macrocycle training (microcyles 2, 16 and 30, which aimed to monitor the adaptations and assimilation to the training loads applied) as follows: 1) measurement of peak torque and maximal work, using isokinetic dynamometry, during concentric contraction of knee flexor and extensor muscles and 2) measurement of JP (lower limbs), using the countermovement vertical jump (CMVJ) performed on a force platform. measure. A 48-hour interval was established between the last training session and the laboratory evaluations. The athletes were instructed to avoid physical activities during this period.

\section{Muscular Strength Evaluation}

MS was measured using the gold standard Biodex System 4 Pro $^{\circledR}$ isokinetic dynamometer (Biodex, New York, NY), which has the highest correlation coefficients for reliability, accuracy, and validity ${ }^{13}$. The players performed five maximal isokinetic contractions for measuring peak torque and maximal work at the speed of $60 \%$ s, during concentric contraction of knee flexor and extensor muscles. Prior to the evaluation, the laterality of the participants was determined, following a procedure established in a previous study ${ }^{14}$. The athletes were then seated on the isokinetic dynamometer with the hips in $80^{\circ}$ flexion. The axis of the instrument was aligned with the knee joint axis. To minimize extraneous movements of the body during contractions of the thigh muscles, straps were secured across the chest, pelvis, and thigh ${ }^{15}$. The center of resistance was located at the distal $1 / 3$ of the 1 eg, $2 \mathrm{~cm}$ above the lateral malleolus of the tibia tarsal joint and then fixed with adjacent straps. The participants were asked to position their arms across their chest. The weight of the limb to be tested was checked by the dynamometer for automatic correction of the parameter values measured to avoid the effect of gravity ${ }^{14}$. The range of motion was between $100^{\circ}$ and $10^{\circ}$, corresponding to $90^{\circ}$ and it was limited to avoid the effect of passive insufficiency of the hamstring muscles, that is, when an antagonist muscle (hamstring muscles) limits the action of an agonist muscle (quadriceps). Prior to the test, the participants performed three submaximal isokinetic contractions to familiarize themselves with the equipment and its operation, as well as the strength needed according to the selected speed ${ }^{16}$. The lower limb used for starting the test was chosen at random. Visual and audio feedback was provided to the subjects ${ }^{14,15}$.

\section{Jump Power Evaluation}

JP was evaluated using a force platform (OR7/6/1000, AMTI, Watertown, MA, USA), which is a high-quality measurement tool developed for jump/power testing ${ }^{17,18}$. Data acquisition and analysis were performed using AMTI NetForce ${ }^{\circledR}$ and BioAnalysis ${ }^{\circledR}$ software (Watertown, MA, USA). The sampling rate was $1000 \mathrm{~Hz}$, and the acquisition time was 8 seconds. Prior to the test, the subjects warmed up, by performing submaximal bilateral jumps on the force platform, followed by three maximal CMVJ series, with rest periods of 30 seconds between series. The athletes were instructed to remain with their hands on their waist and to quickly perform the eccentric phase of each jump (knee angle of approximately $120^{\circ}$ ) and then jump as high as possible, during the concentric phase. Knees and ankles were to remain extended "upon landing"19. During the tests, the participants were verbally encouraged to maximize their jumping abilities.

\section{Statistical analysis}

The data were analyzed by the Sigma-Stat ${ }^{\circledR}$ program, version 2.03 , and are presented as mean \pm standard deviation. The power and strength values were analyzed using the one-way ANOVA and Kruskal-Wallis tests, when applicable. The significance level was set to $\mathrm{p}<0.05$ for all analyses.

\section{Results}

\section{Muscular Strength}

Table 4 shows the values of peak torque (absolute and relative) and maximal work (absolute and relative) for basketball players during different moments of the training macrocycle, obtained from the isokinetic dynamometer software. No significant differences were observed in any of the parameters analyzed in this study when comparing the three test moments (microcycles 2, 16, and 30).

\subsection{Jump Power}

JP parameters (flight time, height, and maximal power) of the basketball athletes were obtained with the use of a force platform during three distinct moments of the macrocycle training (microcycles 2, 16, and 30) and are shown in Table 5 The values of jump height achieved by each athlete during the three moments is seen in Figure 2. No differences were observed when the analyzed parameters were compared. 
Table 4. Values of peak torque and maximal work of basketball players measured by isokinetic dynamometry at the speed of $60^{\circ} / \mathrm{s}$ during concentric contraction of knee flexor and extension in three different moments of macrocycle training.

\begin{tabular}{|c|c|c|c|c|}
\hline Moments & Microcycle 2 & Microcycle 16 & Microcycle 30 & (P) \\
\hline \multicolumn{5}{|c|}{ Knee extension (Dominant limb) } \\
\hline Peak Torque (N.m) & $279.7 \pm 36.5$ & $293.7 \pm 31.9$ & $296.0 \pm 40.0$ & 0.522 \\
\hline Maximal Work (J) & $318.0 \pm 46.8$ & $312.3 \pm 45.6$ & $325.2 \pm 46.8$ & 0.806 \\
\hline Normalized Torque (\%) & $326.2 \pm 36.3$ & $336.5 \pm 37.5$ & $330.1 \pm 37.2$ & 0.719 \\
\hline Normalized Work (\%) & $370.8 \pm 48.6$ & $357.2 \pm 48.0$ & $362.9 \pm 42.0$ & 0.760 \\
\hline \multicolumn{5}{|c|}{ Knee extension (Nondominant limb) } \\
\hline Peak Torque $(\mathrm{N} \cdot \mathrm{m})$ & $268.7 \pm 39.6$ & $271.8 \pm 33.7$ & $272.6 \pm 41.0$ & 0.966 \\
\hline Maximal Work (J) & $295.7 \pm 51.3$ & $294.2 \pm 44.2$ & $291.2 \pm 52.0$ & 0.976 \\
\hline Normalized Torque (\%) & $313.6 \pm 39.8$ & $312.8 \pm 45.7$ & $305.8 \pm 49.5$ & 0.905 \\
\hline Normalized Work (\%) & $344.0 \pm 45.4$ & $337.4 \pm 52.4$ & $325.9 \pm 54.2$ & 0.695 \\
\hline \multicolumn{5}{|c|}{ Knee flexion (Dominant limb) } \\
\hline Peak Torque $(\mathrm{N} \cdot \mathrm{m})$ & $138.5 \pm 19.7$ & $139.8 \pm 21.3$ & $146.9 \pm 19.1$ & 0.585 \\
\hline Maximal Work (J) & $160.0 \pm 25.7$ & $158.6 \pm 30.6$ & $164.4 \pm 21.6$ & 0.868 \\
\hline Normalized Torque (\%) & $161.0 \pm 14.7$ & $159.9 \pm 22.9$ & $164.1 \pm 16.4$ & 0.860 \\
\hline Normalized Work (\%) & $185.9 \pm 20.5$ & $180.8 \pm 31.0$ & $183.9 \pm 22.6$ & 0.879 \\
\hline \multicolumn{5}{|c|}{ Knee flexion (Nondominant limb) } \\
\hline Peak Torque (N.m) & $136.3 \pm 17.6$ & $138.7 \pm 16.7$ & $147.7 \pm 14.9$ & 0.253 \\
\hline Maximal Work (J) & $152.7 \pm 27.0$ & $162.7 \pm 27.2$ & $171.5 \pm 20.1$ & 0.225 \\
\hline Normalized Torque (\%) & $159.1 \pm 18.8$ & $159.0 \pm 20.1$ & $165.5 \pm 16.6$ & 0.655 \\
\hline Normalized Work (\%) & $177.9 \pm 27.4$ & $185.2 \pm 29.3$ & $191.9 \pm 21.3$ & 0.460 \\
\hline
\end{tabular}

Values are presented as mean $\pm \mathrm{SD}$.

Table 5. Characteristics of CMVJ performed by basketball players on a force platform in three different moments of the training macrocycle.

\begin{tabular}{lrrrr}
\hline \multicolumn{1}{c}{ CMVJ Parameters } & Microcycle 2 & Microcycle 16 & Microcycle 30 & Valor P \\
\hline Flight time (s) & $0.588 \pm 0.02$ & $0.581 \pm 0.03$ & $0.594 \pm 0.02$ & 0.609 \\
Height (cm) & $43 \pm 4.27$ & $42 \pm 5.12$ & $43 \pm 4.47$ & 0.524 \\
Maximal Power (W) & $4.302 \pm 729$ & $4.268 \pm 735$ & $4.536 \pm 681$ & 0.629 \\
\hline
\end{tabular}

Values are presented as mean $\pm \mathrm{SD}$.

Fig. 2 Height of countermovement vertical jump (CMVJ) performed by each basketball player during different moments of the training macrocycle.

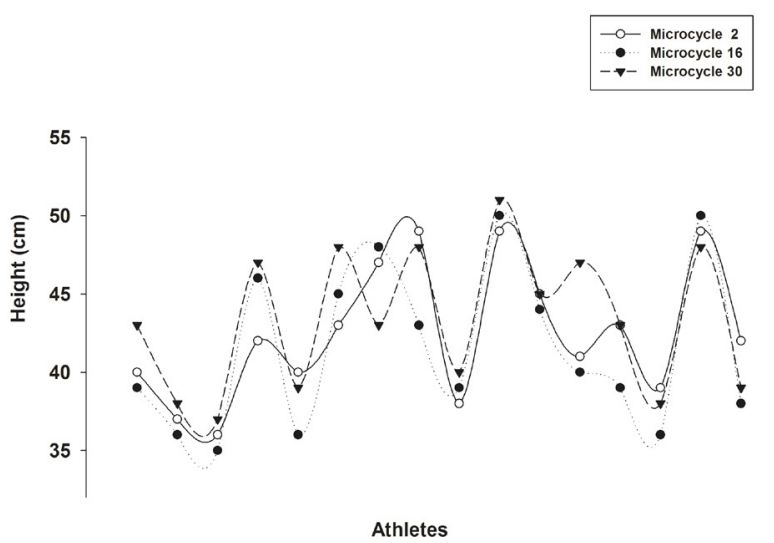

\section{Discussion}

In the present study, MS and JP of basketball players were analyzed in three different periods of a macrocycle training (microcycles 2, 16, and 30) The main finding was that MS and the JP values remained unchanged, during the macrocycle. These results were evidenced by the absence of change in the values of peak torque, maximal work, and performance achieved in CMVJ, assessed by a isokinetic dynamometer and force platform.

The plateau or decrease in the maximal performance of athletes is often associated with an imbalance between the workload and the recovery period ${ }^{20}$. In this context, many studies have demonstrated the use of physical tests to control and evaluate the efficiency of sports training programs $\mathrm{s}^{5,7,8,21-23}$. Strength and power measurements can provide valuable insights into the training state of athletes ${ }^{21}$. In basketball, the 
performance of high-intensity movements is closely associated with the development of strength, sprint, and agility ${ }^{24}$. In fact, the improvement of these motor skills contribute to efficient basketball movements, with and without the ball, that play an important role in the tactical-technical aspects of the game ${ }^{25}$.

However, little is known about training methods and specific adaptations involving basketball athletes and studies that address the use of different periodization models and the performance achieved in physical tests over the course of the macrocycle training, are still incipient. Therefore, for the development of lower limb strength capacity, the present study used resistance training at a bodybuilding gym. The selected exercises (leg press, squat, deadlifting, step-up knee) emphasized the work of the main muscle groups involved in the specific actions of the modality in question. As far as the improvement of the jump power, the method used was the plyometry, with the performance of submaximal and maximum vertical jumps. The training methods used are validated in the literature for increasing MS and JP of lower limbs ${ }^{22,26-29}$. Nevertheless, the training program had no effect on MS and JP of the athletes, when analyzed in different moments of the macrocycle training. Our findings are not consistent with the findings of other studies that have observed increased capacities for muscular strength and jump power in basketball athletes ${ }^{28,29}$. The main reason for this outcome is not clear, and considering the importance in the selection of specific physical tests to evaluate the performance of athletes, it is possible that the results observed in the present study are related to the lack of specificity of the tests used.

The MS, measured by isokinetic dynamometer (considered gold standard in the evaluation of muscle function), has been used more frequently in the evaluation of the imbalances and asymmetries in basketball athletes ${ }^{9,16}$. Thus, the 1 RM test has been suggested, for example, to assess the performance of dynamic lower limb muscle strength in basketball athletes during half-squat exercise ${ }^{30}$.

From the biomechanical point of view, it is necessary to recognize that the jumps performed on the force platform were different from those used in the plyometry sessions and during the technical-tactical training, which could explain the JP results. In fact, a study that investigated the specificity of the tests used to evaluate the jumping power in basketball athletes, concluded that Sargent Jump is highly correlated to the specific movements of this modality. In addition, the manifestation of the maximum vertical jump power does not occur frequently in basketball ${ }^{10}$, which also may explain the observed results. Moreover, it is also possible that the lack of changes in the parameters evaluated is related to the structure of the training loads used in the macrocycle in question. However, the values of peak torque, maximal work, and jump power were maintained by the athletes, when the different moments were compared, suggesting, a possible maintenance of the performance of strength and power of the lower limbs of the athletes, during the macrocycle.

\section{Conclusions}

Our results suggest that the effectiveness of basketball training programs seems to not be related to the performance achieved by athletes on the strength and power tests included in this study. In turn, it is possible that the absence of change in MS and JP values, during the macrocycle, can be assigned to the structure of the training used, i.e, volume, intensity, density and/or specificity of workloads.

\section{Study Limitations}

The study presented limitations mainly regarding the monitoring of other performance markers that would allow establishing correlation with the results obtained. In addition, it was not possible to submit the athletes to different training programs in order to evaluate their effectiveness. Another limitation refers to the size of the sample, which, because it is small, allows us to consider the results found only for the athletes in question.

\section{References}

1. Matthew D, Delextrat A. Heart rate, blood lactate concentration, and time-motion analysis of female basketball players during competition. J Sports Sci. 2009; 27(8): 813-821. doi: 10.1080/02640410902926420

2. Verkhoshanski Y V. Treinamento Desportivo: teoria e metodologia. [Sports Training: theory and methodology]. Porto Alegre: Artemed; 2001.

3. Wen N, Dalbo VJ, Burgos B, Pyne DB, Scanlan AT. Power Testing in Basketball: Current Practice and Future Recommendations. J Strength Cond Res. 2018; Feb 1. doi: 10.1519/JSC.0000000000002459.

4. Matavulj D, Kukolj M, Ugarkovic D, Tihanyi J, Jaric S. Effects of plyometric training on jumping performance in junior basketball players. J Sports Med Phys Fitness 2001; 41(2): 159-164.

5. Boccollini G, Brazzit A, Bonfanti L, Alberti G. Using balance training to improve the performance of youth basketball players. Sport Sci Health 2013; 9(2): 37-42. doi: 10.1007/s11332-013-0143-z

6. Delextrat A, Trochym E, Calleja-González J. Effect of a typical in season-week on strength jump and sprint performance in national-level female basketball players. J Sports Med Phys Fitness 2012; 52(2): 128-136.

7. Chatzinikolaou A, Draganidis D, Avloniti A, Karipidis A, Jamurtas AZ, Skevaki CL, et al. The microcycle of inflammation and performance changes after a basketball match. J Sports Sci. 2014; 32(9): 870-882. doi: 10.1080/02640414.2013.865251

8. Alemdaroğlu U. The relationship between muscle strength, anaerobic performance, agility, sprint ability and vertical jump performance in professional basketball players. J Hum Kinet. 2012 ; 31 : 149-158. doi: 10.2478/v10078-012-0016-6

9. Hadzić V, Erculj F, Bracic M, Dervisević E. Bilateral concentric and eccentric isokinetic strength evaluation of quadriceps and hamstrings in basketball players. Coll Antropol. 2013; 37(3): 859-865.

10. Lamas L. Especificidade do treinamento no Basquetebol: Fatores energéticos e neuromusculares. [Training specificity Basketball: energy and neuromuscular factors]. Rev Mack Ed Fís Esp. 2006; 5(1): 93-106.

11. Gomes AC. Treinamento desportivo - estruturação e periodização [Sports training - structuring and periodization]. Porto Alegre, SC: Artmed; 2002. 
12. Edwards S. The heart rate monitor book. New York: Polar Electro Oy; 1993.

13. Drouin JM, Valovich-mcLeod TC, Shultz SJ, Gansneder BM, Perrin DH. Reliability and validity of the Biodex system 3 pro isokinetic dynamometer velocity, torque and position measurements. Eur J Appl Physiol. 2004; 91(1): 22-29.

14. Rahnama N, Lees A, Bambaecichi E. Comparison of muscle strength and flexibility between the preferred and non-preferred leg in English soccer players. Ergonomics 2005; 48(11-14): 1568-1575. doi: 10.1080/00140130500101585

15. Lund H, Søndergaard K, Zachariassen T, Christensen R, Bülow $P$, Henriksen M, et al. Learning effect of isokinetic measurements in healthy subjects, and reliability and comparability of Biodex and Lido dynamometers. Clin Physiol Funct Imaging 2005; 25(2): 75-82. doi: 10.1111/j.1475-097X.2004.00593.x

16. Zakas A, Mandroukas K, Vamvakoudis E, Christoulas K, Aggelopoulou N. Peak torque of quadriceps and hamstring muscles in basketball and soccer players of different divisions. J Sports Med Phys Fitness 1995; 35(3): 199-205.

17. Requena B, García I, Requena F, Saez-Saez de Villarreal E, Pääsuke M. Reliability and validity of a wireless microelectromechanicals based system (keimove ${ }^{\mathrm{TM}}$ ) for measuring vertical jumping performance. J Sports Sci Med. 2012; 11(1): 115-122.

18. Owen NJ, Watkins J, Kilduff LP, Bevan HR, Bennett M. Development of a criterion method to determine peak mechanical power output in a countermovement jump. J Strength Cond Res. 2014; 28(6):1552-1558. doi: 10.1519/JSC.0000000000000311

19. Cronin JB, Hansen KT. Strength and power predictors of sports speed. J Strength Cond Res. 2005; 19(2): 349-357.

20. Lehmann M, Gastmann U, Petersen KG, Bachl N, Seidel A, Khalaf AN, et al. Training - overtraining: performance, and hormone levels, after a defined increase in training volume versus intensity in experienced middle and long distance runners. Br J Sports Med. 1992; 26(4): 233-242.

21. McGuigan MR, Cormack SJ, Gill ND. Strength and power profiling of athletes: selecting tests and how to use the information for program design. Strength and Conditioning Journal 2013; 35(6): 7-14. doi: 10.1519/SSC.0000000000000011

22. Naclerio F, Faigenbaum AD, Larumbe-Zabala E, Perez-Bibao T, Kang J, Ratamess NA, et al. Effects of different resistance training volumes on strength and power in team sport athletes. J Strength Cond Res. 2013; 27(7): 1832-1840. doi: 10.1519/JSC.0b013e3182736d10

23. Sekulic D, Spasic M, Mirkov D, Cavar M, Sattler T. Genderspecific influences of balance, speed, and power on agility performance. J Strength Cond Res. 2013; 27(3): 802-811. doi: 10.1519/ JSC.0b013e $31825 \mathrm{c} 2 \mathrm{cb} 0$

24. Castagna C, Manzi V, D’Ottavio S, Annino G, Padua E, Bishop D. Relation between maximal aerobic power and the ability to repeat sprints in young basketball players. J Strength Cond Res. 2007; 21(4): 1172-1176.
25. Erčulj F, Blas M, Bračič M. Physical demands on young elite European female basketball players with special reference to speed, agility, explosive strength, and take-off power. J Strength Cond Res. 2010; 24(11): 2970-2978. doi: 10.1519/JSC.0b013e3181e38107

26. Rousanoglou EN, Barzouka KG, Boudolos KD. Seasonal changes of jumping performance and knee muscle strength in under-19 women volleyball players. J Strength Cond Res. 2013; 27(4): 1108-1117. doi: 10.1519/JSC.0b013e3182606e 05

27. Rebaï H, Chtourou H, Zarrouk N, Harzallah A, Kanoun I, Dogui $\mathrm{M}$, et al. Reducing resistance training volume during Ramadan improves muscle strength and power in football players. Int J Sports Med. 2014; 35(5): 432-437. doi: 10.1055/s-0033-1353216

28. Maffiuletti NA, Cometti G, Amiridis IG, Martin A, Pousson M, Chatard JC. The effects of electromyostimulation training and basketball practice on muscle strength and jumping ability. Int J Sports Med. 2000; 21(6): 437-43. doi: 10.1055/s-2000-3837

29. Gonzalez AM, Hoffman JR, Rogowski JP, Burgos W, Manalo E, Weise K, et al. Performance changes in NBA basketball players vary in starters vs. nonstarters over a competitive season. J Strength Cond Res. 2013; 27(3): 611-615. doi: 10.1519/JSC.0b013e31825dd2d9

30. Tsimahidis K, Galazoulas C, Skoufas D, Papaiakovou G, Bassa E, Patikas D, et al. The effect of sprinting after each set of heavy resistance training on the running speed and jumping performance of young basketball players. J Strength Cond Res. 2010;24(8):2102-8. doi:10.1519/JSC.0b013e3181e2e1ed.

\section{Acknowledgements}

The authors declare that they have no conflicts of interest concerning this article and are grateful to the São Paulo Research Foundation (FAPESP-2012/04299-8) for the financial support.

\section{Corresponding author}

Hugo C. D. Souza, Ph.D.

Department of Rehabilitation and Functional Performance. School of Medicine of Ribeirão Preto, University of São Paulo, 14049-900, Ribeirão Preto, SP, Brazil.

E-mail: hugocds@fmrp.usp.br

Manuscript received on June 8, 2018

Manuscript accepted on July 4, 2018

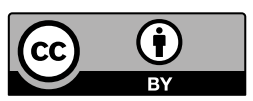

Motriz. The Journal of Physical Education. UNESP. Rio Claro, SP, Brazil - eISSN: 1980-6574 - under a license Creative Commons - Version 3.0 